\title{
Postdischarge Nausea and Vomiting Remains Frequent After LeFort I Osteotomy Despite Implementation of a Multimodal Antiemetic Protocol Effective in Reducing Postoperative Nausea and Vomiting
}

\author{
Carolyn Dicus Brookes, DMD, MD [Chief Resident], Timothy A. Turvey, DDS ${ }^{\dagger}$ [Professor \\ and Chairman], Ceib Phillips, MPH, PhD $\ddagger$ [Professor], Vincent Kopp, MD§ [Professor], and \\ Jay A. Anderson, DDS, MD`[ [Associate Professor] \\ *Department of Oral and Maxillofacial Surgery \\ tDepartment of Oral and Maxillofacial Surgery \\ FDepartment of Orthodontics \\ §Department of Anesthesiology \\ IDepartments of Anesthesiology and Oral and Maxillofacial Surgery
}

\begin{abstract}
Purpose-To assess the prevalence of postdischarge nausea and vomiting (PDNV) after Le Fort I osteotomy with and without the use of a multimodal antiemetic protocol shown to decrease postoperative nausea and vomiting (PONV).
\end{abstract}

\begin{abstract}
Materials and Methods-Consecutive patients undergoing Le Fort I osteotomy with or without additional procedures at a single academic institution formed the intervention cohort for an institutional review board-approved prospective clinical trial with a retrospective comparison group. The intervention cohort was managed with a multimodal antiemetic protocol. The comparison group consisted of consecutive patients who underwent similar surgical procedures at the same institution before protocol implementation. All patients were asked to complete a postdischarge diary documenting the occurrence of nausea and vomiting. Those who completed the diaries were included in this analysis. Data were analyzed with the Fisher exact test and the Wilcoxon rank sum test. A $P$ value less than .05 was considered significant.

Results-Diaries were completed by $85 \%$ of patients in the intervention group (79 of 93) and $75 \%$ of patients in the comparison group (103 of 137). Patients in the intervention $(n=79)$ and comparison $(n=103)$ groups were similar in the proportion of patients with validated risk factors for PDNV, including female gender, history of PONV, age younger than 50 years, opioid use in the postanesthesia care unit (PACU), and nausea in the PACU $(P=.37)$. The prevalence of PDNV was unaffected by the antiemetic protocol. After discharge, nausea was reported by $72 \%$ of patients in the intervention group and $60 \%$ of patients in the comparison group $(P=.13)$ and
\end{abstract}


vomiting was reported by $22 \%$ of patients in the intervention group and $29 \%$ of patients in the comparison group $(P=.40)$.

Conclusion-Modalities that successfully address PONV after Le Fort I osteotomy might fail to affect PDNV, which is prevalent in this population. Future investigation will focus on methods to minimize PDNV.

Postoperative nausea and vomiting (PONV) has been studied extensively. Guidelines have been developed to help minimize PONV, and implementation of a multi-modal protocol has recently been shown to effectively decrease PONV in the orthognathic surgical population. ${ }^{1-3}$ Postdischarge nausea and vomiting (PDNV), although also common, is less well understood. ${ }^{4}$ The recently updated Society for Ambulatory Anesthesia guidelines for the management of PONV emphasize that "PDNV is still a significant problem" despite advances in the prevention of PONV. ${ }^{3}$ In 1 systematic review, $17 \%$ of patients (range, 0 to $55 \%$ ) developed postdischarge nausea (PDN) and 8\% (range, 0 to 16\%) developed postdischarge vomiting $(\mathrm{PDV})^{5}$; another systematic review reported that $32.6 \%$ developed PDN and $14.7 \%$ developed PDV. ${ }^{6}$ PDNV can have a considerable impact on patients, their at-home providers, and the health care system. PDNV can delay resumption of daily activities and can result in readmission. ${ }^{7-9}$ Nausea and vomiting after surgery also can lead to wound complications and stress on home care providers. Intermaxillary elastic traction, hypoesthesia, and facial edema make PDNV particularly distressing after orthognathic surgical procedures.

Despite the negative effect PDNV can exert on recovery, few patients who develop PDNV contact their providers; thus, providers are likely to underestimate this problem. ${ }^{7}$ However, patients place great emphasis on this complication. Patient dissatisfaction has been statistically linked with PONV,${ }^{10,11}$ and evidence shows that fear of PONV eclipses even fear of pain. ${ }^{12}$

Validated risk factors for PDNV have been derived from a prospective multicenter cohort study that assessed nausea and vomiting for 48 hours postoperatively in more than 2,000 patients. These risk factors include female gender, age younger 50 years, history of PONV, opioid administration in the postanesthesia care unit (PACU), and nausea in the PACU. The use of ondansetron intraoperatively, smoking status, and types of surgery were not statistical predictors of PDNV. 3,13

PONV has been shown to occur frequently after orthognathic surgery. ${ }^{14,15}$ A multimodal protocol that decreased prevalence of PONV after Le Fort I osteotomy with or without additional procedures has recently been reported. ${ }^{1}$ The preponderance of studies evaluating modalities to address PONV simply have evaluated effectiveness at discharge from the recovery room or at 24 hours postoperatively; thus, this study also was designed to evaluate PDNV. The purposes of this study were to assess the prevalence of PDNV after Le Fort I osteotomy, with or without additional procedures, and to evaluate the impact of the multimodal protocol on PDNV. The authors hypothesized that the prevalence of PDNV after Le Fort I osteotomy would be high and that it would be decreased by protocol implementation. 


\section{Materials and Methods}

A prospective, institutional review board-approved clinical trial (919.966.3113, University of North Carolina, Chapel Hill) with a retrospective comparison group was registered with ClinicalTrials.gov. (NCT01592708) This study showed a statistical decrease in postoperative nausea (PON) and postoperative vomiting (POV) experienced by patients undergoing Le Fort I osteotomy, with or without additional procedures, after the introduction of a multimodal antiemetic protocol. PDNV was studied in the same patients under the same institutional review board approval to assess the prevalence of PDNV before and after protocol implementation and to test the hypothesis that the protocol also would decrease PDNV. Guidelines of the Declaration of Helsinki were followed. The authors state that the report includes every item in the Strengthening the Reporting of Observational Studies in Epidemiology checklist for cohort observational clinical studies.

Consecutive patients at least 15 years old undergoing Le Fort I osteotomy, with or without additional procedures, from July 2012 through February 2014 were recruited as the intervention cohort receiving a multi-modal antiemetic protocol that is presented in Table 1. ${ }^{1,16-29}$ The protocol was based largely on consensus guidelines. ${ }^{2}$ A detailed rationale for protocol development and a description of inclusion and exclusion criteria have been reported. ${ }^{1}$

The comparison group was comprised of a subset of consecutively enrolled patients with American Society of Anesthesiologists physical status I and II who underwent orthognathic surgery at the same institution from June 2008 through June 2012, before implementation of the multimodal antiemetic protocol. In patients in the comparison group, all anesthetics were at the discretion of the anesthesia care team; the breakdown of anesthetics and analgesics administered is presented in Table 2. The sole inclusion criterion added for the PDNV cohort was diary completion.

A post-orthognathic surgical daily health diary included specific items relating to nausea and vomiting. ${ }^{30,31}$ The comparison cohort completed a 90-day diary, the first 7 days of which were assessed for this study. The prospective cohort completed a 7-day diary. Beginning on postoperative day 1 , patients were asked daily whether they experienced nausea or vomiting. They also were asked to rank their experience of nausea on a 5-point scale, from no trouble or concern (1) to lots of trouble or concern (5). PDN was considered resolved when the patient reported little to no trouble or concern related to nausea. PDV was considered resolved when patients reported no vomiting. Days to resolution of PDN and PDV were determined by the postoperative day on which resolution was reported; thus, 1 day to resolution meant that patients reported little to no trouble or concern related to nausea or no vomiting on postoperative day 1 and thus experienced no PDN or PDV.

Trained research associates described the project to each patient and obtained written consent or assent with parental permission and Health Insurance Portability and Accountability Act authorization to review clinical records. The same research associates provided the diaries to patients before discharge from the hospital. 
Patients returned for routine postoperative visits and were asked to return diaries once completed. If a patient did not return the diary, a research associate provided the patient with a preaddressed envelope to facilitate diary return by mail. Diaries were electronically scanned for data retrieval.

Established patient-related risk factors for PONV, including female gender, nonsmoking status, and history of PONV or motion sickness, were collected from medical records. ${ }^{32}$ Further, validated risk factors for PDNV (female gender, history of PONV, age $<50 \mathrm{yr}$, opioid use in the PACU, and nausea in the PACU) were recorded. ${ }^{13}$ Medical records were reviewed by 2 independent individuals; any differences were resolved by joint record review.

The primary predictor variable was protocol implementation. The primary outcome variables were PDN and PDV. Bivariate comparisons between the intervention and control groups were performed using the Fisher exact test for nominal variables and the exact Wilcoxon rank sum test for continuous variables using SAS 9.1 (SAS Institute, Cary, NC). A $P$ value less than .05 was considered statistically significant.

\section{Results}

Diaries were completed by $85 \%$ of patients in the intervention group $(n=79)$ and $75 \%$ of patients in the comparison group $(\mathrm{n}=103)$. The total intervention $(\mathrm{n}=93)$ and comparison $(\mathrm{n}=137)$ groups were similar in gender $(P=.29)$, race $(P=.85)$, age $(P=.75)$, proportion of patients with known risk factors for PONV (including female gender, nonsmoking status, history of PONV or motion sickness, and history of migraine headaches; $P=.34$ ), percentage undergoing bimaxillary surgery (60\% in each group), and percentage for whom surgical time was longer than 180 minutes $(P=.51)$. The only statistically significant difference between those who completed diaries and those who did not according to these traits was that there were fewer female patients who did not complete diaries in the comparison cohort (47 vs 71\%; $P=.011$; Table 3). Despite this difference, those who completed diaries in the intervention and comparison groups were similar in known risk factors for PONV (Fig 1).

The patients in each group who completed diaries also were compared for validated risk factors for PDNV. Fifty-eight percent of patients in the intervention group and $71 \%$ of patients in the comparison group were female $(P=.08)$. No patients were older than 50 years. History of PONV was noted for $8 \%$ of patients in the intervention group and for $10 \%$ of patients in the comparison group $(P=.62)$. Opioids were administered in the PACU to more patients in the intervention group (70\%) than to patients in the comparison group (35\%; $P<.0001)$. However, fewer patients in the intervention group $(5 \%)$ experienced nausea in the PACU than did patients in the comparison group $(20 \% ; P=.003)$. Nonetheless, the proportion of patients with a given number of validated risk factors was similar in each group $(P=.37$; Fig 2$)$.

PON was significantly decreased by the antiemetic protocol in the intervention versus comparison cohort ( 23 vs $71 \% ; P<.01)$. Likewise, POV was significantly decreased in the 
intervention versus comparison cohort ( $10 \mathrm{vs} 30 \% ; P<.01)$. The difference between cohorts was similar to that observed in the previous PONV report comparing 93 and 137 patients. ${ }^{1}$

Diary analysis for PDNV showed that $72 \%$ of patients who received the antiemetic protocol versus $60 \%$ of patients in the comparison group reported PDN $(P=.09)$ and that $22 \%$ of patients in the intervention group versus $29 \%$ of patients in the comparison group reported $\operatorname{PDV}(P=.24$; Fig 3$)$.

Median day on which nausea resolved was day 4 (interquartile range [IQR], days 1 to 7) for the intervention group and day 2 (IQR, days 1 to 7) for the comparison group. Median day on which patients reported no vomiting was day 1 (IQR, day 1) for the intervention group and day 1 (IQR, days 1 to 2 ) for the comparison group. There was no statistically significant difference in time to resolution of PON $(P=.24)$ or POV $(P=.22)$ between the 2 groups.

\section{Discussion}

The authors hypothesized that the prevalence of PDNV would be high after Le Fort I surgery and that the prevalence would be decreased by the antiemetic protocol. The prevalence of PDNV was indeed very high in this patient population. However, although this multimodal antiemetic protocol statistically decreased PON and POV, ${ }^{1}$ it had no statistical impact on PDNV. There was a trend toward slightly more PDN that lasted slightly longer in the intervention group, but a trend toward more PDV in the comparison group. Sample size limits the ability to detect relevance, but these findings support the concept that PONV and PDNV are different entities that must be addressed separately. $3,7,13$

PDN lasted longer in the present series (intervention group: mean, 4.2 days; standard deviation [SD], 2.83 days; comparison group: mean, 3.74 days; SD, 2.96 days) than in another large series (mean, 1.7 days; $\mathrm{SD}, 1.3$ days). ${ }^{13}$

When attempting to address PDNV, risk factor assessment is crucial so high-risk patients can be identified and managed appropriately. According to the simplified risk score for PDNV by Apfel et al, ${ }^{13}$ PDNV will be experienced by approximately 10, 20, 30, 50, 60, and $80 \%$ of patients with $0,1,2,3,4$, or 5 of the following risk factors: female gender, age younger than 50 years, history of PONV, opioid administration in the PACU, and nausea in the PACU. Sensitivity and specificity of the risk score are 65 to $70 \%{ }^{3}$ Most patients in each group in this series had no more than 2 risk factors. Prevalence of PDNV was higher than expected based on the simplified risk score, underscoring that much remains unknown about PDNV.

Although the role of surgery type remains controversial in PONV,${ }^{3}$ the results of this study suggest that Le Fort I osteotomy could be an unrecognized high-risk surgery type for PDNV as an independent risk factor or as a result of confounding factors. The important dietary alterations required after orthognathic surgery, including Le Fort I osteotomy, could contribute to the prolonged duration of PDNV in this population. Unfortunately, the present sample is underpowered to identify risk factors for PDNV; however, additional investigation into risk factors for PDNV is important because better understanding of these risk factors 
will enhance providers' ability to decrease this unpleasant postoperative problem shown to delay resumption of normal daily activities in the postoperative period. ${ }^{7}$

The protocol was initially designed to target PONV; however, several strategies implemented in the intervention cohort also have been linked to decreasing PDNV. A systematic review found that less PDNV is seen after total intravenous anesthetic (TIVA) than after use of volatile agents ${ }^{33}$; recent consensus guidelines highlight this. ${ }^{3}$ In contrast, White et $\mathrm{al}^{34}$ reported more PDNV in patients managed with TIVA than in those managed with a volatile agent. However, only those managed with a volatile agent received dolasetron; no other antiemetic strategies were used in this group of high-risk patients. Another systematic review found that dexamethasone statistically decreases PDN (relative risk [RR], 0.55), but not PDV. ${ }^{6}$ Methylprednisolone, the steroid administered to patients in the 2 groups in this study, has been shown to decrease late PONV extending to 24 hours and beyond. $^{35,36}$

Ondansetron $4 \mathrm{mg}$ has been shown to statistically decrease PDN (RR, 0.77) and PDV (RR, 0.63). ${ }^{6}$ Combination therapy has been shown to decrease PDNV more than single agents. ${ }^{6,37}$ Opioid-sparing analgesia is another strategy posited to decrease PDNV. ${ }^{38}$ Scopolamine has been shown to have equivalent effect to ondansetron and droperidol on nausea and vomiting in the 72 hours after surgery ${ }^{39}$ and to decrease nausea and vomiting further at 24 and 48 hours after surgery when given in addition to ondansetron. ${ }^{40}$

Despite administration of a TIVA with combination antiemetic therapy that included steroids, scopolamine, ondansetron and opioid-sparing analgesia, there was no impact on PDNV in the intervention group.

Many medications currently used to prevent PONV have a relatively short half-life, which can limit their utility in the prevention of PDNV. One potential solution to this issue is administration of scheduled oral ondansetron postoperatively; this intervention, alone or in conjunction with administration of dexamethasone, has been shown to decrease PDNV, increase patient satisfaction, and improve quality of functional living. ${ }^{41,42}$ Other investigators have turned toward newer agents with longer half-lives. Among these are palonosetron, a serotonin (5-HT3) receptor antagonist with a 40-hour half-life and unique allosteric inhibition of 5-HT3 receptors in addition to positive cooperative binding. ${ }^{43}$ Palonosetron shows promise in the management of nausea and vomiting in the first 72 hours after surgery $3,38,44-46$ and has a good safety profile: although corrected QT(QTc) prolongation has been reported with palonosetron in conjunction with sevoflurane,${ }^{47}$ it is much less apt to cause QTc prolongation than are the other 5-HT3 receptor antagonists. ${ }^{44,46,48}$

Aprepitant and other neurokinin-1 receptor antagonists (NK1-RAs) also might play a role in the management of PDNV. NK1-RAs are competitive inhibitors of the receptors for substance $\mathrm{P}$, which is linked to emesis; these agents show particular promise in the decrease of vomiting. NK1-RAs are nonsedating and do not cause QTc prolongation, although aprepitant does inhibit CYP3A4 in a dose-dependent fashion. ${ }^{3,38,45,49-51}$ A recent trial reported no difference in PDNV from 24 to 48 hours postoperatively in patients receiving 
aprepitant versus placebo ${ }^{52}$; although it could be an important adjunct in the prevention of PDNV, further investigation into aprepitant's impact in the postdischarge period is indicated.

Acupoint stimulation of "pericardium 6," located on the wrist, is low risk and might decrease PONV and PDNV; acupressure and other noninvasive acupoint stimulation techniques seem to be as effective as invasive acupoint stimulation. ${ }^{3,45,53}$

Limitations of this study include the small sample and use of a retrospective comparison group. As a result of the retrospective comparison group and slightly different exclusion criteria, confounding variables could have been introduced. The retrospective comparison group offered the advantage of comparison with existing treatment at the discretion of the anesthesia care team rather than with a prescribed alternative to the multimodal protocol. Practitioners should use caution in generalizing these findings to other populations.

This study found a high incidence of PDNV in patients undergoing Le Fort I osteotomy, with or without additional procedures, despite implementation of an antiemetic protocol that effectively decreased PONV in the same patients. Because PDNV is important for patient safety, productivity, and satisfaction, additional investigation into methods to minimize this postoperative complication in the orthognathic surgical population is indicated, particularly because more of these procedures are performed on an outpatient basis.

\section{Acknowledgments}

This research was funded by National Institutes of Health grant R01 DE 005215.

\section{References}

1. Dicus Brookes C, Berry J, Rich J, et al. Multimodal protocol reduces post-operative nausea and vomiting in patients undergoing Le Fort I osteotomy. J Oral Maxillofac Surg. 2015; 73:324. [PubMed: 25443378]

2. Gan TJ, Meyer TA, Apfel CC, et al. Society for Ambulatory Anesthesia guidelines for the management of postoperative nausea and vomiting. Anesth Analg. 2007; 105:1615. [PubMed: 18042859]

3. Gan TJ, Diemunsch P, Habib AS, et al. Consensus guidelines for the management of postoperative nausea and vomiting. Anesth Analg. 2014; 118:85. [PubMed: 24356162]

4. Chinnappa V, Chung F. Post-discharge nausea and vomiting: An overlooked aspect of ambulatory anesthesia? Can J Anaesth. 2008; 55:565. [PubMed: 18840585]

5. Wu CL, Berenholtz SM, Pronovost PJ, et al. Systematic review and analysis of postdischarge symptoms after outpatient surgery. Anesthesiology. 2002; 96:994. [PubMed: 11964610]

6. Gupta A, Wu CL, Elkassabany N, et al. Does the routine prophylactic use of antiemetics affect the incidence of postdischarge nausea and vomiting following ambulatory surgery? Anesthesiology. 2003; 99:488. [PubMed: 12883424]

7. Carroll NV, Miederhoff P, Cox FM, et al. Postoperative nausea and vomiting after discharge from outpatient surgery centers. Anesth Analg. 1995; 80:903. [PubMed: 7726432]

8. Gold BS, Kitz DS, Lecky JH, et al. Unanticipated admission to the hospital following ambulatory surgery. JAMA. 1989; 262:3008. [PubMed: 2810644]

9. Philip BK. Patients' assessment of ambulatory anesthesia and surgery. J Clin Anesth. 1992; 4:355. [PubMed: 1389187]

10. Lehmann M, Monte K, Barach P, et al. Postoperative patient complaints: A prospective interview study of 12,276 patients. J Clin Anesth. 2010; 22:13. [PubMed: 20206846] 
11. Myles PS, Williams DL, Hendrata M, et al. Patient satisfaction after anaesthesia and surgery: Results of a prospective survey of 10,811 patients. Br J Anaesth. 2000; 84:6. [PubMed: 10740539]

12. Macario A, Weinger M, Carney S, et al. Which clinical anesthesia outcomes are important to avoid? The perspective of patients. Anesth Analg. 1999; 89:652. [PubMed: 10475299]

13. Apfel CC, Phillip B, Cakmakkaya OS, et al. Who is at risk for post-discharge nausea and vomiting after ambulatory surgery? Anesthesiology. 2012; 117:475. [PubMed: 22846680]

14. Silva AC, O'Ryan F, Poor DB. Postoperative nausea and vomiting (PONV) after orthognathic surgery: A retrospective study and literature review. J Oral Maxillofac Surg. 2006; 64:1385. [PubMed: 16916674]

15. Phillips, C.; Dicus Brookes, C.; Rich, J., et al. Post-operative nausea and vomiting following orthognathic surgery. Int J Oral Maxillofac Surg. http://dx.doi.org/10.1016/j.ijom.2015.01.006

16. Kranke P, Morin AM, Roewer N, et al. The efficacy and safety of transdermal scopolamine for the prevention of postoperative nausea and vomiting: A quantitative systematic review. Anesth Analg. 2002; 95:133. [PubMed: 12088957]

17. Kopp VJ, Mayer DC, Shaheen NJ. Intravenous erythromycin promotes gastric emptying prior to emergency anesthesia. Anesthesiology. 1997; 87:703. [PubMed: 9316982]

18. Itoh Z, Nakaya M, Suzuki T, et al. Erythromycin mimics exogenous motilin in gastrointestinal contractile activity in the dog. Am J Physiol Gastrointest Liver Physiol. 1984; 247:G688.

19. Barkun AN, Bardou M, Kuipers EJ, et al. International consensus recommendations on the management of patients with nonvariceal upper gastrointestinal bleeding. Ann Int Med. 2010; 152:101. [PubMed: 20083829]

20. Barkun AN, Bardou M, Martel M, et al. Prokinetics in acute upper GI bleeding: A meta-analysis. Gastrointest Endosc. 2010; 72:1138. [PubMed: 20970794]

21. Apfel CC, Korttila K, Abdalla M, et al. IMPACT Investigators. A factorial trial of six interventions for the prevention of postoperative nausea and vomiting. N Engl J Med. 2004; 350:2441. [PubMed: 15190136]

22. Tramer M, Moore A, McQuay H. Propofol anaesthesia and postoperative nausea and vomiting: Quantitative systematic review of randomized controlled studies. Br J Anesth. 1997; 78:247.

23. Tramer MR, Fuchs-Buder T. Omitting antagonism of neuromuscular blockade: Effect on postoperative nausea and vomiting and risk of residual paralysis. A systematic review $\mathrm{Br} \mathbf{J}$ Anaesth. 1999; 82:379.

24. Habib AS, Gan TJ. The effectiveness of rescue antiemetics after failure of prophylaxis with ondansetron or droperidol: A preliminary report. J Clin Anesth. 2005; 17:62. [PubMed: 15721732]

25. Scuderi PE, James RL, Harris L, et al. Multimodal antiemetic management prevents early postoperative vomiting after outpatient laparoscopy. Anesth Analg. 2000; 91:1408. [PubMed: 11093990]

26. Precious DS, Multari J, Finley GA, et al. A comparison of patient-controlled and fixed schedule analgesia after orthognathic surgery. J Oral Maxillofac Surg. 1997; 55:33. [PubMed: 8994466]

27. Rodriguez RF, Castillo JM, Castillo MDP, et al. Codeine/acetaminophen and hydrocodone/ acetaminophen combination tablets for the management of chronic cancer pain in adults: A 23day, prospective, double-blind, randomized, parallel-group study. Clin Ther. 2007; 29:581. [PubMed: 17617281]

28. Mukherjee K, Esuvaranathan V, Streets C, et al. Adenotonsillectomy in children: A comparison of morphine and fentanyl for peri-operative analgesia. Anaesthesia. 2001; 56:1193. [PubMed: 11736779]

29. Claxton AR, McGuire G, Chung F, et al. Evaluation of morphine versus fentanyl for postoperative analgesia after ambulatory surgical procedures. Anesth Analg. 1997; 84:509. [PubMed: 9052292]

30. Phillips C, Blakey G III. Short-term recovery after orthognathic surgery: A medical daily diary approach. Int J Oral Maxillofac Surg. 2008; 37:892. [PubMed: 18768296]

31. Phillips C, Blakey G III, Jaskolka M. Recovery after orthognathic surgery: Short-term healthrelated quality of life outcomes. J Oral Maxillofac Surg. 2008; 66:2110. [PubMed: 18848110]

32. Apfel CC, Laara E, Koivuranta M, et al. A simplified risk score for predicting postoperative nausea and vomiting: Conclusions from cross-validations between two centers. Anesthesiology. 1999; 91:693. [PubMed: 10485781] 
33. Gupta A, Stierer T, Zuckerman R, et al. Comparison of recovery profile after ambulatory anesthesia with propofol, isoflurane, sevoflurane and desflurane: A systematic review. Anesth Analg. 2004; 98:632. [PubMed: 14980911]

34. White H, Black RJ, Jones M, et al. Randomized comparison of two anti-emetic strategies in highrisk patients undergoing day-case gynaecological surgery. Br J Anesth. 2007; 98:470.

35. Miyagawa Y, Ejiri M, Kuzuya T, et al. Methylprednisolone reduces postoperative nausea in total knee and hip arthroplasty. J Clin Pharm Ther. 2010; 35:679. [PubMed: 21054459]

36. Weren M, Demeere JL. Methylprednisolone vs dexamethasone in the prevention of postoperative nausea and vomiting: A prospective, randomized, double-blind, placebo controlled trial. Acta Anaesthesiol Belg. 2008; 59:1. [PubMed: 18468010]

37. White PF, O'Hara JF, Roberson CR, et al. POST-OP Study Group. The impact of current antiemetic practices on patient outcomes: A prospective study on high-risk patients. Anesth Analg. 2008; 107:452. [PubMed: 18633023]

38. Melton MS, Klein SM, Gan TJ. Management of postdischarge nausea and vomiting after ambulatory surgery. Curr Opin Anesthesiol. 2011; 24:612.

39. White PF, Tang J, Song D, et al. Transdermal scopolamine: An alternative to ondansetron and droperidol for the prevention of postoperative and postdischarge emetic symptoms. Anesth Analag. 2007; 104:92.

40. Gan TJ, Sinha AC, Kovac AL, et al. A randomized, double-blind, multicenter trial comparing transdermal scopolamine plus ondansetron to ondansetron alone for the prevention of postoperative nausea and vomiting in the outpatient setting. Anesth Analg. 2009; 108:1498. [PubMed: 19372328]

41. Pan PH, Lee SC, Harris LC. Antiemetic prophylaxis for postdischarge nausea and vomiting and impact on functional quality of living during recovery in patients with high emetic risks: A prospective, randomized, double-blind comparison of two prophylactic antiemetic regimens. Anesth Analg. 2008; 107:429. [PubMed: 18633020]

42. Gan TJ, Franiak R, Reeves J. Ondansetron orally disintegrating tablet versus placebo for the prevention of postdischarge nausea and vomiting after ambulatory surgery. Anesth Analg. 2002; 94:1199. [PubMed: 11973189]

43. Kloth DD. New pharmacologic findings for the treatment of PONV and PDNV. Am J Health Syst Pharm. 2009; 66:S11. [PubMed: 19106333]

44. Apfel CC, Jukar-Rao SS. Is palonosetron also effective for opioid-induced and postdischarge nausea and vomiting? Br J Anaesth. 2012; 108:371. [PubMed: 22337957]

45. George E, Hornuss C, Apfel CC. Neurokinin-1 and novel serotonin antagonists for postoperative and postdischarge nausea and vomiting. Curr Opin Anesth. 2010; 23:714.

46. Kovac AL, Eberhart L, Kotarski J, et al. Palonosetron 04-07 Study Group. A randomized, doubleblind study to evaluate the efficacy and safety of three different doses of palonosetron versus placebo in preventing postoperative nausea and vomiting over a 72-hour period. Anesth Analg. 2008; 107:439. [PubMed: 18633021]

47. Min JJ, Yoo Y, Kim TK, et al. Intravenous palonosetron increases the incidence of QTc prolongation during sevoflurane general anesthesia for laparotomy. Korean J Anesthesiol. 2013; 65:397. [PubMed: 24363841]

48. Gonullu G, Demircan S, Demirag MK, et al. Electrocardiographic findings of palonosetron in cancer patients. Support Care Cancer. 2012; 20:1435. [PubMed: 21773677]

49. Apfel CC, Malhotra A, Leslie JB. The role of neurokinin-1 receptor antagonists for the management of postoperative nausea and vomiting. Curr Opin Anesthesiol. 2008; 21:427.

50. Gan TJ, Apfel CC, Kovac A, et al. Aprepitant-PONV Study Group. A randomized, double-blind comparison of the NK1 antagonist, aprepitant, versus ondansetron for the prevention of postoperative nausea and vomiting. Anesth Analg. 2007; 104:1082. [PubMed: 17456656]

51. [Accessed March 30, 2014] DailyMed: ALOXI-Palonosetron hydrochloride capsule, gelatin coated. Available at: http://dailymed.nlm.nih.gov/dailymed/lookup.cfm?setid=bd06f321bb42-4748-92f3-d59626b540e0 
52. Jung WS, Kim YB, Park HY, et al. Oral administration of aprepitant to prevent postoperative nausea in highly susceptible patients after gynecological laparoscopy. J Anesth. 2013; 27:396. [PubMed: 23224788]

53. Lee A, Fan LT. Stimulation of the wrist acupuncture point P6 for preventing postoperative nausea and vomiting. Cochrane Database Syst Rev. 2009; 2:CD003281. [PubMed: 19370583] 
A

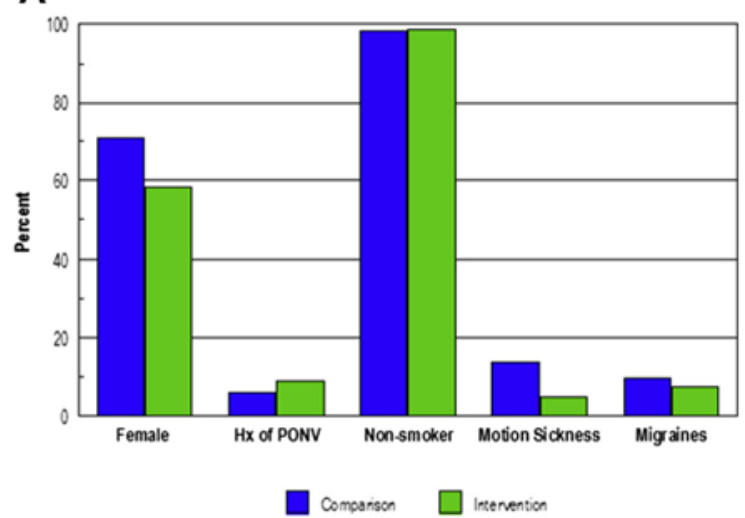

B



Comourison $\square$ intervenion

Figure 1.

Classic risk factors for PONV (including female gender, nonsmoking status, history of PONV or motion sickness, and history of migraine headaches) in diary responders in comparison versus intervention cohort. $A$, Comparison of number of patients with each risk factor. $B$, Comparison of number of patients with a given number of risk factors. Hx, history; PONV, postoperative nausea and vomiting 
A

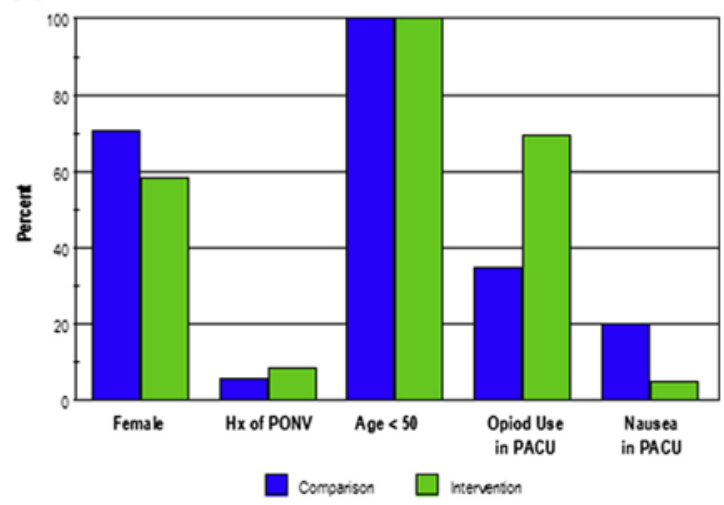

B

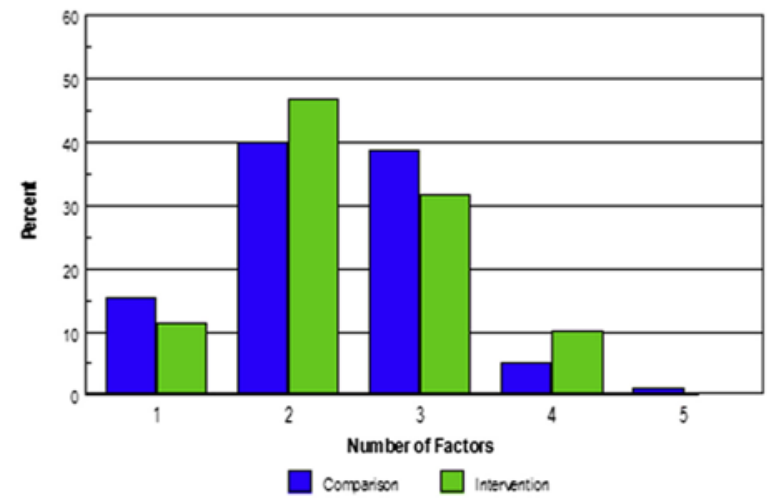

Figure 2.

Validated risk factors for PDNV (including female gender, history of PONV, age $<50 \mathrm{yr}$, opioid use in the PACU, and nausea in the PACU) for diary responders in comparison versus intervention cohort. $A$, Comparison of number of patients with each risk factor. $B$, Comparison of number of patients with a given number of risk factors. PACU, postanesthesia care unit; PDNV, postdischarge nausea and vomiting 


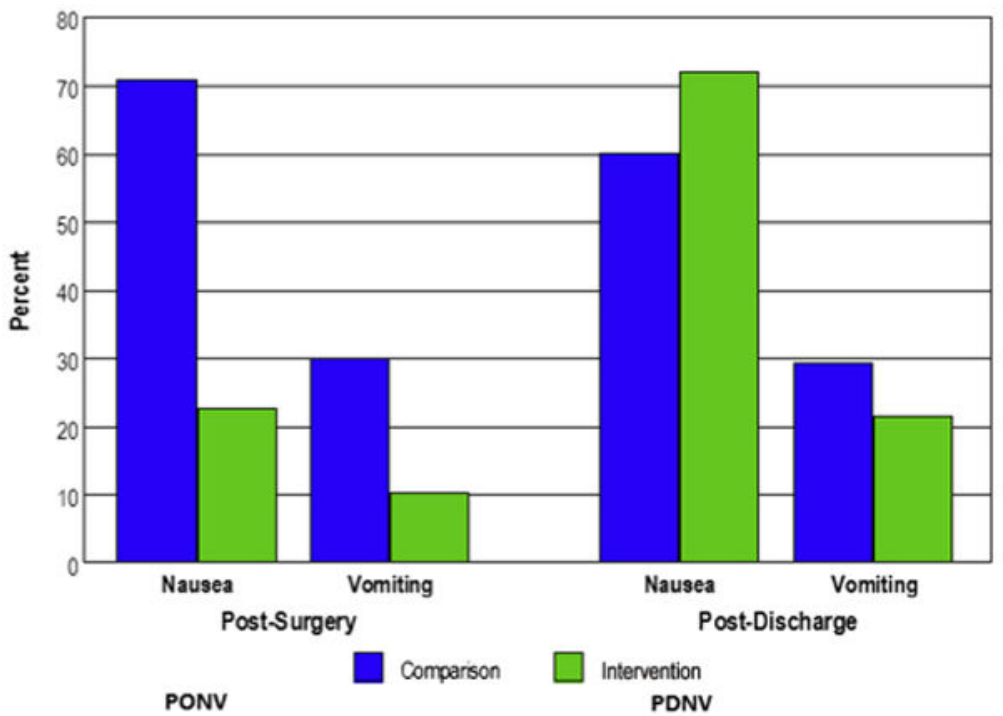

Figure 3.

Comparison of prevalence of PONV and PDNV in diary responders in the comparison and intervention groups. PDNV, postdischarge nausea and vomiting; PONV, postoperative nausea and vomiting. 
Table 1

\section{Summary of Multimodal Protocol Used for Patients in the Intervention Group ${ }^{1}$}

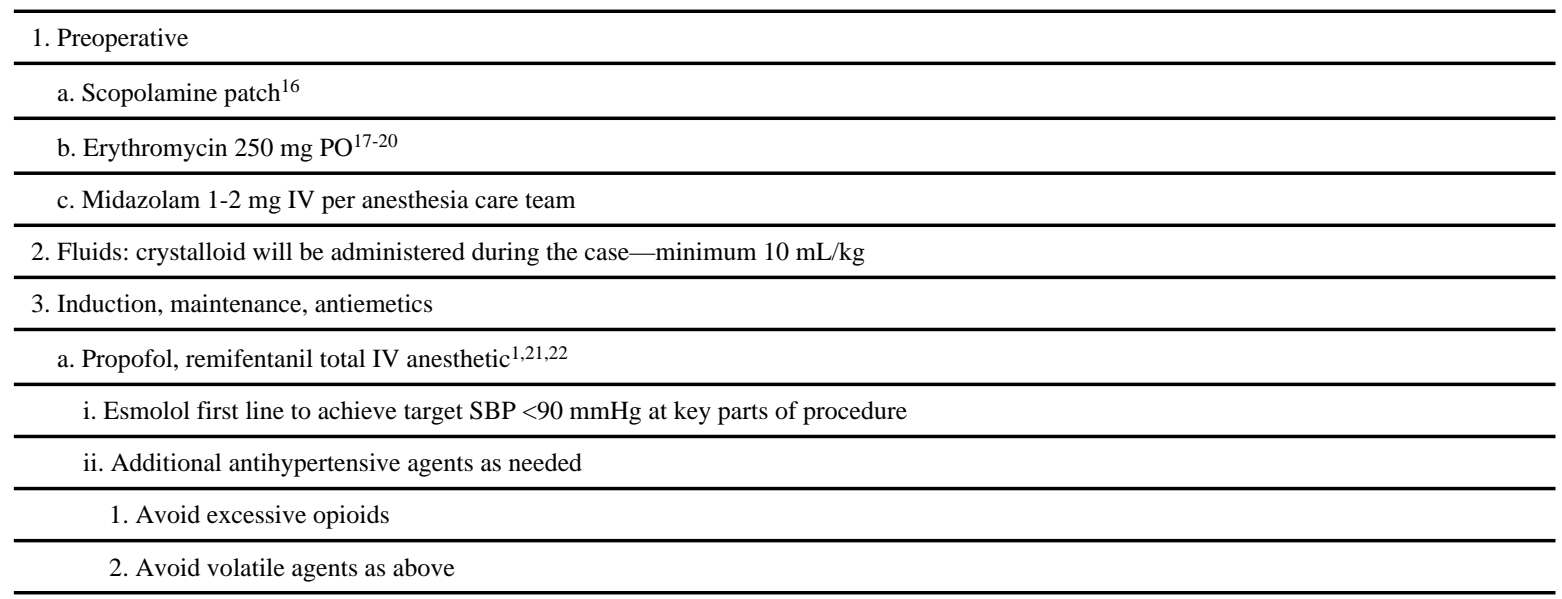

b. Neostigmine: avoid if possible (paralysis not surgically necessary); if used, limit to maximum $2.5 \mathrm{mg}$ unless patient safety dictates otherwise ${ }^{1,23}$

c. Steroids per surgeon preference; typically Solu-Medrol $125 \mathrm{mg}$ IV at beginning of case

d. Droperidol $0.625 \mathrm{mg}$ IV near end of case ${ }^{1,21}$

e. Ondansetron $4 \mathrm{mg}$ IV near end of case $\mathrm{c}^{1,21}$

f. Surgeons to evacuate gastric contents with a nasogastric tube at end of case

4. Analgesia in OR

a. Ketorolac $30 \mathrm{mg}$ IV at end of case ${ }^{1}$

b. No morphine; fentanyl preferred, hydromorphone if necessary

5. PACU or floor

a. Rescue medications for nausea or emesis ${ }^{1,24}$

i. Promethazine $6.25-25 \mathrm{mg} I V$, PO, or PR

ii. Dexamethasone $4 \mathrm{mg}$ IV (PACU only)

iii. Diphenhydramine $25-50 \mathrm{mg}$ IV or PO

iv. Ondansetron $4 \mathrm{mg}$ IV or 4-8 $\mathrm{mg}$ ODT (if $\Varangle 6 \mathrm{~h}$ after first dose)

b. Fluids: continue crystalloid in PACU until goal of $\geq 25 \mathrm{~mL} / \mathrm{kg}$ is reached $\mathrm{d}^{1,25}$

c. Analgesia1 ${ }^{4,26-29}$

i. No morphine; fentanyl preferred, hydromorphone if necessary

ii. No codeine; hydrocodone or acetaminophen preferred; oxycodone if necessary

iii. Ketorolac $30 \mathrm{mg}$ IV q6h until conversion to PO analgesics, then scheduled ibuprofen $600 \mathrm{mg}$ q $6 \mathrm{~h}^{1}$

d. Prokinetics: repeat erythromycin $250 \mathrm{mg}$ PO 8 hours after first dose in OR

e. Steroids: redosed per surgeon preference

Abbreviations: IV, intravenous; ODT, orally disintegrating tablet; OR, operating room; PACU, postanesthesia care unit; PO, per oral; PR, per rectum; q6h, every 6 hours; SBP, systolic blood pressure. 
Table 2

Anesthetics Used in Retrospective Group

\begin{tabular}{|c|c|}
\hline \multicolumn{2}{|r|}{ 1. Anesthetic agents } \\
\hline & a. Volatile agents, $98 \%$ \\
\hline & b. Nitrous oxide, $54 \%$ \\
\hline & 2. Fluids: per anesthetist preference \\
\hline & 3. Analgesia \\
\hline & a. Morphine, $69 \%$ \\
\hline & b. Codeine, $61 \%$ \\
\hline & 4. Antiemetic: ondansetron, $97 \%$ \\
\hline
\end{tabular}

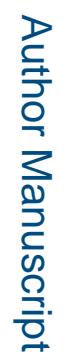

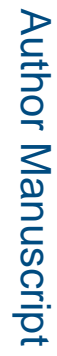

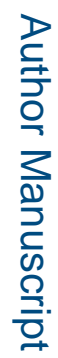

J Oral Maxillofac Surg. Author manuscript; available in PMC 2016 July 01. 


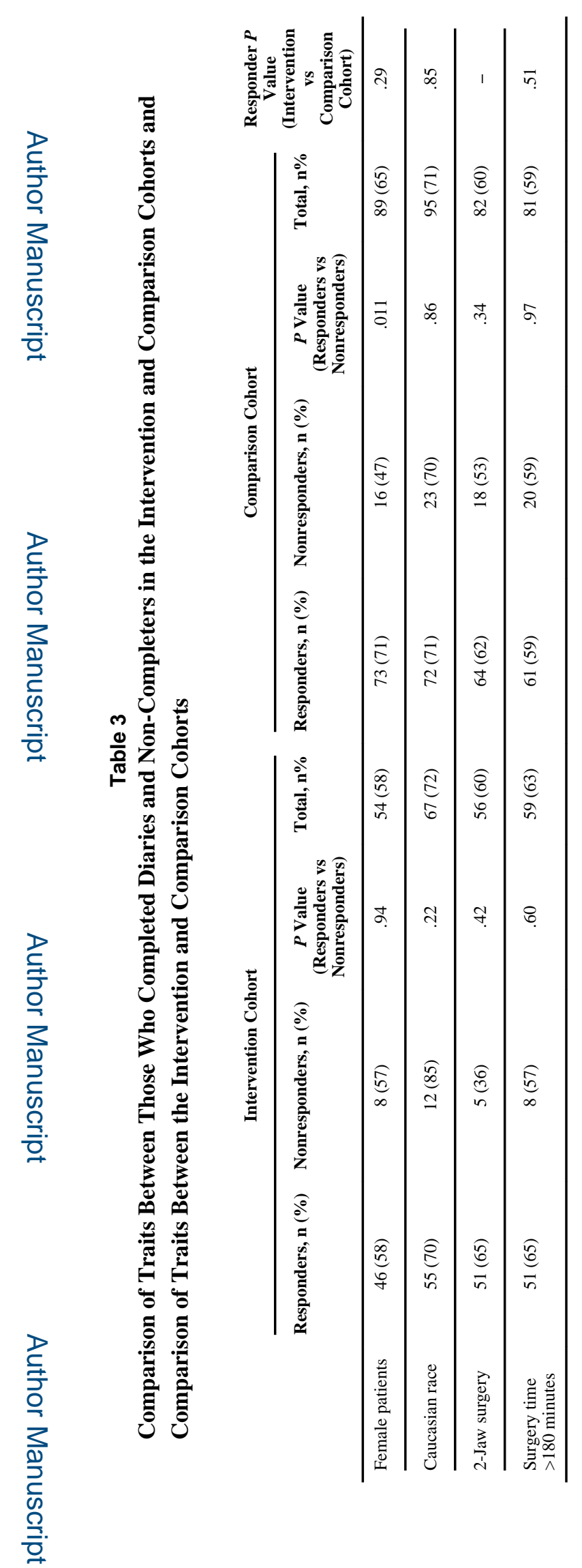

J Oral Maxillofac Surg. Author manuscript; available in PMC 2016 July 01. 